\title{
The spectrum of the Dirac operator on coset spaces with homogeneous gauge fields
}

\author{
Brian P. Dolan \\ Dept. of Mathematical Physics, NUI, Maynooth, Ireland and \\ School of Theoretical Physics \\ Dublin Institute for Advanced Studies \\ 10 Burlington Rd., Dublin 8, Ireland \\ E-mail: bdolan@thphys.may.ie
}

\begin{abstract}
The spectrum and degeneracies of the Dirac operator are analysed on compact coset spaces when there is a non-zero homogeneous background gauge field which is compatible with the symmetries of the space, in particular when the gauge field is derived from the spin-connection. It is shown how the degeneracy of the lowest Landau level in the recently proposed higher dimensional quantum Hall effect is related to the Atiyah-Singer index theorem for the Dirac operator on a compact coset space.
\end{abstract}

KeYwords: Field Theories in Higher Dimensions, Differential and Algebraic Geometry. 


\section{Contents}

1. Introduction 1

2. Symmetric spaces 3

3. Non-symmetric spaces 11

4. Conclusions 13

A. Metric and connection on $G / H$

B. Spectrum of the Dirac operator on $C P^{2} \quad 16$

G. Index theorem on $\mathrm{SU}(3) / \mathrm{U}(1) \times \mathrm{U}(1)$

\section{Introduction}

There has long been a fruitful interplay between condensed matter physics and field theory in particle physics, many concepts that were first developed in the former later being applied to the latter and vice versa. The quantum Hall effect [1] has attracted the interest of many high energy theorists, not least because the fractional QHE exhibits collective excitations which mimic a fractional electric charge, but also because there are deeper connections between the Hall effect and string theory [2]. Recently Zhang and Hu proposed a higher dimensional analogue of the quantum Hall effect, on $S^{4}$ [3], based on Haldane's description of the Hall effect on $S^{2}$ with a magnetic monopole at the centre, [⿴囗十. Zhang and Hu's idea was developed further in [5] and extended to complex projective spaces in [5]. The connection between the higher dimensional quantum Hall effect and string theory was analysed in [7].

The higher dimensional quantum Hall effect involves a generalisation of the Landau problem to particles moving on a compact coset space $G / H$, in the presence of a background gauge field: such as a $\mathrm{U}(1)$ monopole on $C P^{n}$ or a homogeneous $\mathrm{SU}(2)$ instanton field on $S^{4}$. A common ingredient of the these analyses is the calculation of the degeneracy of the ground state for particles moving in a homogeneous background, i.e. a background field which has the symmetry of the isometry group $G$. In previous works, and in this paper also, the gauge group will be restricted to be the holonomy group $H$ (or a factor group of same if $H$ is a product of smaller groups).

In [3, [6] the degeneracy of the ground state was calculated using group theory: the non-relativistic hamiltonian for a spinless particle moving in a homogeneous background field involves the quadratic Casimirs of the groups $G$ and $H$ and the allowed states involve 
irreducible representations of $G$ that contain pre-ordained representations of $H$. The dimension of the representation of $G$ corresponding to the ground state is identified with the degeneracy of the ground state.

It is common in discussions of the quantum Hall effect to ignore the electron's spin. Zhang and $\mathrm{Hu}$ treated scalar particles satisfying the exclusion principle, as did Karabali and Nair: this is perfectly justified when the Zeeman splitting is large enough that transitions between spin states can be ignored and the ground state is effectively isolated from the next highest spin state. Nevertheless one is tempted to ask what is the rôle of electron spin in the higher dimensional quantum Hall effect, and it will be argued here that there is an important quantitative relic of the Fermionic nature of the particles in the degeneracy of the ground state, over and above the trivial consequences of the exclusion principle. It is shown in section 2 that the degeneracies calculated in [3, 4, 5], for $S^{4}, S^{2}$ and $C P^{n}$ respectively, are related to the index of the Dirac operator for Fermions moving in the appropriate background field: in fact the degeneracy is the number of zero-modes of the Dirac operator and, generically, this is the modulus of the index. Furthermore the ground state wave-functions, the higher dimensional analogues of the Laughlin wave-functions, are precisely the zero-modes of the Dirac operator.

We do not have to look far to discover the reason for this - the square of the Dirac operator is nothing other than the hamiltonian for a non-relativistic Fermion moving in a static background field,

$$
(i \not D)^{2}=-D_{\alpha} D^{\alpha}+\frac{R}{4} 1-\frac{i}{2} F_{\alpha \beta} \gamma^{\alpha \beta},
$$

where $R$ is the Ricci scalar and the last term represents the Zeeman splitting. (There is an extra term on the right hand side of (1.1) if the spin connection involves torsion, this equation must therefore be modified for non-symmetric coset spaces with torsion as considered in section 3.) Thus a non-relativistic particle moving in a static background magnetic field is an example of supersymmetric quantum mechanics with a self-dual pre-potential [8]. Since the Dirac operator is hermitean, the eigenvalues of the hamiltonian (1.1) are positive semidefinite and a zero-mode requires exact cancellation of all three terms on the right hand side. It is shown in sections 2 and 3 that, for specific homogeneous background fields (analogues of monopole and instanton fields on $S^{2}$ and $S^{4}$ ) all three terms on the right hand side of (1.1) are mutually commuting and so can be simultaneously diagonalised so that all spin components decouple from each other. The Dirac laplacian $\Delta=-D_{\alpha} D^{\alpha}$ is itself a positive operator on a compact space with positive curvature, so a zero-mode of the Dirac operator, if one exists, requires a cancellation of the lowest eigenvalue of the laplacian with the lowest eigenvalue of the sum of curvature and Zeeman terms on the right hand side of (1.1). In fact, for the homogeneous background fields that are considered here, the eigenvalues of (1.1) can be determined purely in terms of certain quadratic Casimirs of the isometry group $G$ and the holonomy group $H$ and are given by equation (2.15) or, more generally, (3.9).

Even without calculating the full spectrum it is possible to find the representation of $G$ with the lowest eigenvalue for any Fermion in a given representation of the gauge group. If the chosen representation of the gauge group allows for a zero-mode of the Dirac operator 
then the dimension of the representation corresponding to the lowest eigenvalue generically gives the number of zero-modes. Since this calculation involves only the lowest eigenvalue of the Zeeman and the curvature terms, which are fixed in advance, the Fermionic nature of the particles can be ignored and the problem reduces to choosing the correct representations of $G$ to scan in minimising the laplacian. This is precisely what was done in [3] and [6.

The net result is that the number of zero-modes of the Dirac operator, for Fermions in a given representation of the gauge group, can be calculated simply from a knowledge of the quadratic Casimirs of $G$ and the decompositions of its representations under $H \mapsto G$. This generalises the results of [3] and [6] to the quantum hall effect on any coset space $G / H$ with compact Lie groups $G$ and $H$.

Of course an analysis of the spectrum of the Dirac operator is of intrinsic interest, even without reference to the quantum Hall effect. In particular it is of obvious importance in Kaluza-Klein theories and and string theory.

The layout of the paper is as follows. In section 2 the case of symmetric spaces $G / H$ is treated in detail and it is shown how the eigenvalues of the Dirac operator in the presence of a homogeneous background gauge field can be expressed in terms of quadratic Casimirs $C_{2}(G)$ and $C_{2}(H)$. The examples of $S^{2}, S^{4}$ and $C P^{2}$ are worked out and compared to known results. Section 3 extends the analysis to non-symmetric spaces with torsion and the example of $\mathrm{SU}(3) / \mathrm{U}(1) \times \mathrm{U}(1)$ is treated in detail - this space is of interest in string theory where it is arises in the context of seven dimensional spaces with $G_{2}$ holonomy and their conical singularities [9]. Section 1 gives a summary of the results. Some technical details are relegated to three appendices: appendix A reviews aspects of the geometry of homogeneous spaces used in the text. Appendix B presents the spectrum of the Dirac operator on $C P^{2}$, in the presence of a homogeneous background $\mathrm{SU}(2) \times \mathrm{U}(1)$ gauge field. Appendix $\mathrm{C}$ presents a standard analysis of the Atiyah-Singer index theorem on $\mathrm{SU}(3) / \mathrm{U}(1) \times \mathrm{U}(1)$ for comparison with the results of section 3 .

\section{Symmetric spaces}

Consider the Dirac operator for a Fermion moving on a $d$-dimensional compact space, without boundary, in the presence of a background gauge field:

$$
i \not=i \gamma^{\alpha} D_{\alpha}=i e^{m}{ }_{\alpha} \gamma^{\alpha}\left(\partial_{m}+\frac{1}{4} \omega_{m, \alpha \beta} \gamma^{\alpha \beta}+i A_{m}^{i} t_{i}\right),
$$

where $\omega_{\alpha \beta}=\omega_{\alpha \beta, m} d x^{m}$ is the spin connection, $A^{i}=A_{m}^{i} d x^{m}$ the gauge connection and $t_{i}$ are generators of the gauge group $(\alpha, \beta=1,2, \cdots, d$ are orthonormal indices and $m=1,2, \cdots, d$ is a co-ordinate index). The $\gamma$-matrices satisfy the usual Clifford algebra,

$$
\left\{\gamma^{\alpha}, \gamma^{\beta}\right\}=2 \delta^{\alpha \beta}, \quad \text { with } \quad \gamma^{\alpha \beta}:=\frac{1}{2}\left[\gamma^{\alpha}, \gamma^{\beta}\right]
$$

and $e^{m}{ }_{\alpha}$ are $d$-beins for the metric. The curvature and field strength follow from

$$
\left[D_{\alpha}, D_{\beta}\right]=i F_{\alpha \beta}^{i} t_{i}+\frac{1}{4} R_{\alpha \beta \gamma \delta} \gamma^{\gamma \delta} .
$$


For a torsion free connection squaring the Dirac operator gives

$$
(i D)^{2}=\Delta+\frac{R}{4} \mathbf{1}-\frac{i}{2} F_{\alpha \beta} \gamma^{\alpha \beta},
$$

with $\Delta=-D^{\alpha} D_{\alpha}$ the Dirac laplacian. We shall refer to $\Delta+\frac{R}{4} \mathbf{1}$ as the kinetic energy and $-\frac{i}{2} F_{\alpha \beta} \gamma^{\alpha \beta}=-\frac{i}{2} F_{\alpha \beta}^{i} t_{i} \gamma^{\alpha \beta}$ as the Zeeman energy. The laplacian here is the laplacian acting on spinors, including the spin and the gauge connection, so its spectrum depends on both the metric and the background field.

On a coset space $G / H$, with $G$ and $H$ compact groups, it is natural to use the $G$ invariant metric, for which the generators of $G$ are Killing vectors and the holonomy group is $H \subseteq \mathrm{SO}(d)$. Furthermore we shall consider background gauge fields which are compatible with the isometries, in the sense that Lie transport of the field strength $F$ by a Killing vector $K$ generates a gauge transformation,

$$
\mathcal{L}_{K} F=g^{-1} F g
$$

where $g \in \mathcal{G}$, the group of gauge transformations. In particular this will be the case if we identify the gauge group with the holonomy group and the gauge connection with the spin connection - the details of this identification are given in appendix A. (A variation on this is if the holonomy group factorises into simple groups and $\mathrm{U}(1)$ factors. When this is the case the gauge group can be taken to be one of the factors. For example this is the situation for the homogeneous $\mathrm{SU}(2)$ instanton on $S^{4}$, where $S^{4}=\mathrm{SO}(5) / \mathrm{SO}(4)$ and, at the level of the algebras, $H=\mathrm{SU}(2) \times \mathrm{SU}(2)$ so we can take the gauge group to be just $\mathrm{SU}(2)$.)

Let $t_{A}$ be the generators of the isometry group $G$, with $\left[t_{A}, t_{B}\right]=i f_{A B} t_{C}$, and $t_{i}$ the generators of the holonomy group $H$. Then the curvature 2-forms of a $G$-invariant metric for a symmetric space can be taken to be (see appendix A),

$$
R_{\beta}^{\alpha}=\frac{1}{2} R_{\beta \gamma \delta}^{\alpha} \mathrm{e}^{\gamma} \wedge \mathrm{e}^{\delta}=\frac{1}{2} f_{\beta i}^{\alpha} f_{\gamma \delta}^{i} \mathrm{e}^{\gamma} \wedge \mathrm{e}^{\delta} .
$$

Identifying the gauge connection with the spin connection gives rise to the field strength,

$$
F^{i}=\frac{1}{2} F_{\alpha \beta}^{i} \mathrm{e}^{\alpha} \wedge \mathrm{e}^{\beta}=\frac{1}{2} f_{\alpha \beta}^{i} \mathrm{e}^{\alpha} \wedge \mathrm{e}^{\beta} .
$$

For a symmetric space the Riemann tensor is co-variantly constant and this means that the above field strength is co-variantly constant,

$$
D_{\alpha} F_{\beta \gamma}^{i}=0 .
$$

In particular the laplacian commutes with the Zeeman term in the hamiltonian.

With this choice of background field the commutator (2.3) simplifies,

$$
\left[D_{\alpha}, D_{\beta}\right]=i f_{\alpha \beta}^{i}\left\{\left(\mathbf{1} \otimes t_{i}\right)-\frac{i}{4} f_{i \gamma \delta}\left(\gamma^{\gamma \delta} \otimes \mathbf{1}\right)\right\} .
$$

Now

$$
T_{i}:=-\frac{i}{4} f_{i \gamma \delta} \gamma^{\gamma \delta}
$$


are a representation of the gauge group (which may be reducible, in general),

$$
\left[T_{i}, T_{j}\right]=i f_{i j}{ }^{k} T_{k}
$$

so

$$
\left[D_{\alpha}, D_{\beta}\right]=f_{\alpha \beta}^{i} D_{i}
$$

with

$$
D_{i}:=i\left\{\left(\mathbf{1} \otimes t_{i}\right)+\left(T_{i} \otimes \mathbf{1}\right)\right\}
$$

being the generators of $H$ in the tensor product representation of $t_{i}$ with the spinor representation $T_{i}$. This allows the laplacian to be expressed as the difference of quadratic Casimirs,

$$
\Delta=-D_{\alpha} D_{\alpha}=-D_{A} D_{A}+D_{i} D_{i}=C_{2}(G, \cdot)-C_{2}\left(H, D_{i}\right) .
$$

For spinors in a given representation $t_{i}$ of the gauge group $C_{2}\left(H, D_{i}\right)$ in this expression is always calculated in the fixed representation (2.13), which in general involves reducible representations of $G$, while the representations used in $C_{2}(G, \cdot)$ range over all irreducible representations of $G$ than contain (2.13). In particular the cross-term $-2 t_{i} \otimes T_{i}$ from $\left(D_{i}\right)^{2}$ in (2.14) exactly cancels the Zeeman energy in (2.4) and, as described in appendix A, the second order Casimir for the representation $T_{i}$ is related to the Ricci scalar by $C_{2}\left(H, T_{i}\right)=$ $R / 8$. The eigenvalues of the square of the Dirac operator (2.4) can then be expressed purely in terms of quadratic Casimirs:

$$
E=C_{2}(G, \cdot)-C_{2}\left(H, t_{i}\right)+\frac{R}{8} \mathbf{1}
$$

This construction will now be illustrated with some examples.

(i) $S^{2} \cong \mathrm{SO}(3) / \mathrm{SO}(2)$. This was the geometry originally studied by Haldane in the context of the quantum Hall effect [四]. The isometry group is generated by the algebra of $\mathrm{SU}(2)$

$$
\left[t_{A}, t_{B}\right]=i \epsilon_{A B}^{C} t_{C}
$$

and we are free to choose $t_{3}$ to generate the U(1) holonomy. Formula (A.9) of appendix A gives

$$
R_{\alpha \beta}=\frac{1}{2} \epsilon_{\alpha \beta 3} \epsilon^{3 \gamma \delta} \mathrm{e}^{\gamma} \wedge \mathrm{e}^{\delta}
$$

so

$$
R_{12}=\mathrm{e}^{1} \wedge \mathrm{e}^{2}
$$

are the curvature 2-forms for a sphere of unit radius. Also

$$
F^{3}=\mathrm{e}^{1} \wedge \mathrm{e}^{2}
$$

is the field strength if a magnetic monopole at the centre of the sphere. Actually this corresponds to a monopole of charge 2 , since

$$
\frac{1}{2 \pi} \int_{S^{2}} \mathrm{e}^{1} \wedge \mathrm{e}^{2}=2
$$


is the Chern class of the tangent bundle (which is equal to the Euler characteristic). In general we can put a monopole of any integral charge at the centre of the sphere

$$
F^{3}=\frac{M}{2} \mathrm{e}^{1} \wedge \mathrm{e}^{2} .
$$

(Alternatively we can work with a monopole of charge 2 and consider Fermions of any half-integral charge in this background.)

Choosing $\gamma^{1}=\sigma^{1}$ and $\gamma^{2}=\sigma^{2}$, with $\sigma^{1}$ and $\sigma^{2}$ Pauli matrices, we have

$$
\frac{i}{2} F_{\alpha \beta}^{3} \gamma^{\alpha \beta}=i F_{12}^{3}\left(i \sigma^{3}\right)=-\frac{M}{2}\left(\begin{array}{cc}
1 & 0 \\
0 & -1
\end{array}\right) .
$$

The Ricci scalar for a sphere of unit radius is 2 , so equation (2.4) gives

$$
(i \not)^{2}=\Delta+\frac{1}{2} \mathbf{1}+\frac{M}{2}\left(\begin{array}{cc}
1 & 0 \\
0 & -1
\end{array}\right) \text {. }
$$

For positive $M$ this indicates that there are spin down zero-modes of the Dirac operator if

$$
\Delta+\frac{1}{2}=\frac{M}{2}
$$

while for negative $M$ there are spin up zero-modes if

$$
\Delta+\frac{1}{2}=-\frac{M}{2} .
$$

There are of course no zero-modes for $M=0$ as required by Lichnerowicz theorem.

In this example $t_{i}$ of (2.13) is just a number, $M / 2$, and $T_{i}$ is $\sigma_{3} / 2$ so

$$
D_{3}=i\left(\begin{array}{cc}
\frac{M+1}{2} & 0 \\
0 & \frac{M-1}{2}
\end{array}\right) \quad \Rightarrow \quad D_{3} D_{3}=-\left(\begin{array}{cc}
\left(\frac{M+1}{2}\right)^{2} & 0 \\
0 & \left(\frac{M-1}{2}\right)^{2}
\end{array}\right) \text {. }
$$

The eigenvalues of the laplacian (2.14) are therefore

$$
\Delta_{j}=j(j+1)-\left(\frac{M \pm 1}{2}\right)^{2}
$$

as discussed in [3], so eigenvalues of (2.23) are

$$
E_{j}=\frac{(2 j+1)^{2}-M^{2}}{4}
$$

which can also be obtained directly from (2.15). For $M=0$ this reproduces the well-known result that the spectrum of the Dirac operator is linear in angular momentum (see e.g. [10]).

For $M \neq 0$ the representations $j$ of SU(2) that appear in a harmonic expansion of $\Delta$ are restricted to those that contain the $\mathrm{U}(1)$ representation of charge $\frac{M \pm 1}{2}$, i.e. $j=\frac{|M|-1}{2}+k$ with $k$ a non-negative integer,

$$
E_{j}=k(k+|M|)
$$


There are zero-modes for $k=0$, and $j_{\min }=\frac{M-1}{2}$ for positive $M$ or $-\left(\frac{M+1}{2}\right)$ for negative $M$. In either case the degeneracy of the ground state is

$$
d\left(j_{\min }\right)=2 j_{\min }+1=|M|,
$$

which is the number of zero-modes of the Dirac operator. Note the shift of $j_{\text {min }}$ away from $|M|$ by $1 / 2$, due to the intrinsic spin of the Fermion.

The degeneracy (2.30) relates to the Atiyah-Singer index theorem which states that the the index of the Dirac operator is minus the first Chern class [11],

$$
\nu=\nu_{+}-\nu_{-}=-\frac{1}{2 \pi} \int_{S^{2}} F^{3}=-M
$$

where $\nu_{+}$is the number of positive chirality zero-modes and $\nu_{-}$the number of negative chirality zero-modes. Indeed the ground state wave-functions in [四 for the integer quantum Hall effect, spherical analogues of the Laughlin wave-functions, are precisely these zeromodes. The case $|M|=1$ corresponds to $j_{\min }=0$, in this case the gauge connection exactly cancels the spin connection for the relevant chirality and single zero-mode of the Dirac operator is a constant spinor.

The above calculation can be represented graphically using Young tableaux, which will be useful in more complicated situations to follow. The fundamental of $\mathrm{SU}(2)$ decomposes as

$$
\mathrm{SU}(2) \rightarrow \mathrm{U}(1) \quad \mathbf{2} \rightarrow \mathbf{1}_{1}+\mathbf{1}_{-1} .
$$

Denoting $\mathbf{1}_{1}$ by $\otimes$ and $\mathbf{1}_{-1}$ by $\emptyset$ the $(p+1)$-dimensional irreducible representation of $\mathrm{SU}(2)$ contains

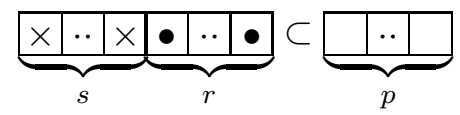

with $p=r+s$. Fixing the $\mathrm{U}(1)$ charge to be $Q$ constrains $s-r=Q$ so $p=2 r+Q$. The ground state energy for a Fermion in this background can now be found by minimising $\Delta$, since all the other terms in the energy are constants for fixed $Q$, that is by minimising

$$
\frac{p}{2}\left(\frac{p}{2}+1\right)=r(r+1)+\frac{Q}{2}(2 r+1)+\frac{Q^{2}}{4} .
$$

If $Q>0$ this is minimised by $r=0$, so $p=Q$ and the degeneracy of the ground state is $Q+1$. If $Q<0$ it is minimised by $r=p$ and then $p=-Q$, so the degeneracy is $-Q+1$. In either case the ground state has $p=|Q|$ and the degeneracy is $|Q|+1$. Clearly $|Q|=2 j_{\text {min }}$ and the $\mathrm{U}(1)$ charge $Q$ is not just the monopole charge $M$, but includes a shift to account for the intrinsic spin of the Fermion, $|Q|=|M|-1$.

This method, using representation theory to describe the kinetic energy and calculate the degeneracy of the ground state, was used in [6]: though in that reference the particles were treated as scalars so there was no intrinsic spin - there was therefore no Zeeman energy to make the total ground state energy vanish and no shift in the charge to account for the intrinsic spin of the particles. The technique is however applicable to both the laplacian for scalars and the square of the Dirac operator because, for a given gauge background and representation, they only differ by constants. It has the advantage of avoiding an explicit calculation of the full eigenvalue spectrum of the Dirac operator. 
(ii) $S^{4} \cong \mathrm{SO}(5) / \mathrm{SO}(4)$. The next example, $S^{4}$, was the case studied in the first paper on the higher dimensional quantum Hall effect, [3]. In this case the algebra of the holonomy group is $\mathrm{SU}(2) \times \mathrm{SU}(2)$ and we can take the gauge group to be just one $\mathrm{SU}(2)$ factor. The Riemann tensor can be split into self-dual and anti-self-dual parts and these correspond to the curvatures arising from the two $\mathrm{SU}(2)$ factors of the holonomy group. Choosing, for example, the self-dual $\mathrm{SU}(2)$ factor the resulting $\mathrm{SU}(2)$ background gauge field is the homogeneous instanton of charge one, which has $\mathrm{SO}(5)$ symmetry on $S^{4}$ [12] (this paper was published a little after the BPST instanton [13, but the techniques are very enlightening and highlight the analogy with the Wu-Yang monopole - Yang calls this homogeneous instanton configuration a non-abelian monopole).

Representations of $\mathrm{SO}(5)$ can be labelled by two integers $p$ and $q$ with $p \geq q$. The second order Casimir and dimension are given by

$$
C_{2}(p, q)=\frac{p^{2}+q^{2}}{2}+2 p+q
$$

and

$$
d(p, q)=\frac{1}{6}(p+q+3)(p-q+1)(p+2)(q+1)
$$

respectively.

Now suppose we have a particle on $S^{4}$ in the representation $I$ of $\mathrm{SU}(2)$ in the background of a homogeneous instanton. Demanding that an $\mathrm{SO}(5)$ irreducible representation contains the $I$ of $\mathrm{SU}(2)$ implies 12 .

$$
p-q=2 I
$$

and so

$$
C_{2}(q+2 I, q)=q^{2}+q(2 I+3)+2 I^{2}+4 I .
$$

The Ricci scalar for the unit four-sphere is $R=12$ so the eigenvalues (2.15) of $(i \not)^{2}$ for a Fermion in the representation $J$ of the gauge group are thus

$$
E=q^{2}+q(2 I+3)+2 I^{2}+4 I-2 J(J+1)+\frac{3}{2} .
$$

(The factor of two in front of the gauge Casimir $J(J+1)$ here is due to the fact that the Dirac operator is non-chiral, the holonomy group is $\mathrm{SU}(2) \times \mathrm{SU}(2)$, and both chiralities couple to the gauge group in the same way.) The total isospin $I$ is a combination of the gauge isospin $J$ and the intrinsic spin of the Fermion, $I=J \pm 1 / 2$, so the energy levels are labelled by the integer $q$ and

$$
\begin{aligned}
& E_{+}(q)=q^{2}+q(2 I+3)+2(2 I+1) \\
& E_{-}(q)=q^{2}+q(2 I+3)
\end{aligned}
$$

both with degeneracies

$$
d(2 I+q, q)=\frac{1}{6}(2 q+2 I+3)(2 I+1)(q+2 I+2)(q+1) .
$$


The spectrum of the Dirac operator on $S^{4}$ has already been studied in the literature. With $J=0$, so $I=1 / 2$, (2.40) reduces to

$$
E_{+}(q)=(q+2)^{2}
$$

The eigenvalues of the Dirac operator itself are therefore linear in $q$ and reproduce the result of [10], with $q=j-1 / 2$ in their notation.

Zero-modes of the Dirac operator require $J \neq 0$ and $I=J-1 / 2$ so that $q=0$ has vanishing eigenvalue, $E_{-}(0)=0$. The degeneracy of this ground state is

$$
d(2 I, 0)=\frac{1}{6}(2 I+3)(2 I+2)(2 I+1)
$$

and these are precisely the degeneracies found in [3] for the ground state of the higher dimensional quantum Hall effect on $S^{4}$. This can be related to the index of the Dirac operator. Recall that the $\mathrm{SU}(2)$ instanton in the $J$ representation can be represented by the field strength

$$
F=F^{i} t_{i} \quad \text { with } \quad F^{i}=\mathrm{e}^{i} \wedge \mathrm{e}^{4}+\frac{1}{2} \epsilon^{i j k} \mathrm{e}^{j} \wedge \mathrm{e}^{k}
$$

and $\sum_{i} t_{i} t_{i}=J(J+1) \mathbf{1}, t_{i}$ being $(2 J+1) \times(2 J+1)$-dimensional matrices (see [1]] for example). The index of the Dirac operator for a Fermion in the $J$-representation of the gauge group $\mathrm{SU}(2)$ is then

$$
\nu=\frac{1}{2(2 \pi)^{2}} \operatorname{tr} \int_{S^{4}} F \wedge F=\frac{1}{4 \pi^{2}} \operatorname{tr}\left(t_{i} t_{i}\right) \int_{S^{4}} \mathrm{e}^{1} \wedge \mathrm{e}^{2} \wedge \mathrm{e}^{3} \wedge \mathrm{e}^{4}=\frac{2}{3}(2 J+1) J(J+1),
$$

since the unit $S^{4}$ has volume $\int_{S^{4}} \mathrm{e}^{1} \wedge \mathrm{e}^{2} \wedge \mathrm{e}^{3} \wedge \mathrm{e}^{4}=8 \pi^{2} / 3$. This agrees with the degeneracy of the ground state $(2.43)$ if

$$
I=J-\frac{1}{2}
$$

The representation $I$ again includes the intrinsic spin of the Fermion: $I$ is in the tensor product of the $J$ with the $1 / 2$ in (2.13). Thus $J=1 / 2$ can give $I=0$ and the single zero-mode of the Dirac operator for a Fermion in the fundamental representation of SU(2) is a constant spinor on $S^{4}$ because the gauge connection again exactly cancels the spin connection in this case. The analogues on $S^{4}$ of the Laughlin-Haldane wave-functions on $S^{2}$ are constructed in [3] by taking anti-symmetrised products of the zero-modes of the Dirac operator in the instanton background with $I=1 / 2$.

(iii) $C P^{2} \cong \mathrm{SU}(3) / \mathrm{U}(2)$. Our final example of a symmetric space is $C P^{2}$. This is not a spin manifold, there is a topological obstruction to defining spinors on this space, but spinors can exist if they are coupled to an appropriate background gauge field [14]. The gauge group is taken to be $\mathrm{SU}(2) \times \mathrm{U}(1)$ and the representations are labelled by the isospin $I$ and the hypercharge $Y$. Representations of $\mathrm{SU}(3)$ which contain the $(I, Y)$ representation of $\mathrm{SU}(2) \times \mathrm{U}(1)$ are easily found. Under $\mathrm{SU}(3) \rightarrow \mathrm{SU}(2) \times \mathrm{U}(1)$ the $\mathbf{3}$ and $\overline{\mathbf{3}}$ decompose as

$$
\mathbf{3} \rightarrow \mathbf{2}_{1}+\mathbf{1}_{-2} \quad \text { and } \quad \overline{\mathbf{3}} \rightarrow \overline{\mathbf{2}}_{-1}+\mathbf{1}_{2} .
$$


Let $\otimes=\mathbf{2}_{1}, \boldsymbol{\bullet}=\mathbf{1}_{-2}, \underset{\mathrm{x}}{\mathrm{x}}=\mathbf{1}_{2}$ and $\dot{\mathrm{x}}=\mathbf{2}_{-1}$. A general irreducible representation of $\mathrm{SU}(3)$ can be labelled by two integers $p$ and $\bar{p}$ and it contains

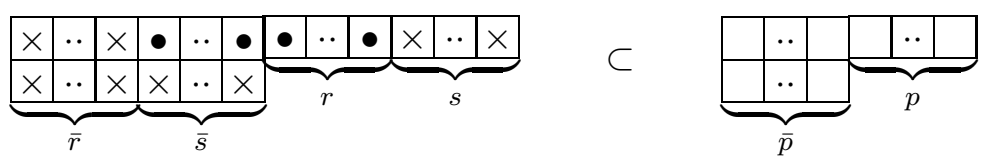

with $r+s=p, \bar{r}+\bar{s}=\bar{p}$. The second order Casimir and dimension are

$$
C_{2}(p, \bar{p})=\frac{1}{3}(p(p+3)+\bar{p}(\bar{p}+3)+p \bar{p})
$$

and

$$
d(p, \bar{p})=\frac{1}{2}(p+\bar{p}+2)(p+1)(\bar{p}+1)
$$

respectively. Furthermore there are constraints

$$
(s-\bar{s})-2(r-\bar{r})=Y \quad \text { and } \quad \frac{s+\bar{s}}{2}=I
$$

for $(p, \bar{p})$ to contain $(I, Y)$. This means that the allowed states are labelled by two integers, as first noted in [6]. Note that $Y$ is odd if $I$ is half-integral and even if $I$ is integral.

The full spectrum of the square of the Dirac operator is given in appendix B, here we shall concentrate on the zero-modes. There are two cases to consider: $|Y| \geq 2 I$ and $|Y| \leq 2 I$ :

- For $|Y|>2 I$ equations $(\mathrm{B.7})$ and $(\mathrm{B.8})$ indicate that there are zero-modes with degeneracy

$$
d(0,0)=\frac{1}{8}(2 I+1)(|Y|-2 I+2)(|Y|+2 I+4)
$$

- For $|Y|<2 I$ equations $(\overline{\mathrm{B} .13})$ and $(\overline{\mathrm{B} .16})$ indicate that there are zero-modes with degeneracy

$$
d(0,0)=\frac{1}{4}(I+1)(2 I+2+Y)(2 I+2-Y)
$$

For $Y=0$ equation 2.53) agrees with the degeneracy of the lowest Landau level for a scalar field derived in [6. Although (2.52) differs from the corresponding expression for scalars in $[6]$, it agrees for $|Y| \rightarrow \infty$, which is the limit used in their analysis.

But now equation (2.52) can be compared with known results for the Dirac index on $C P^{2}$. For $I=0$ we have a $\mathrm{U}(1)$ bundle with $Y$ even and

$$
d(0,0)=\frac{(|Y|+2)(|Y|+4)}{8},
$$

while the index is [14

$$
\nu=\frac{(Q+1)(Q+2)}{2},
$$

with $Q$ the $\mathrm{U}(1)$ charge of the Fermion, including a spin contribution. So $Q>0$ requires $Y=2 Q$, while $Q \leq-3$ requires $Y=2 Q+6 . Q=0$ implies $Y=0$ and is the so-called $\operatorname{spin}^{c}$ structure, where the spin exactly cancels the monopole charge. For the particular cases $Q=-1$ and $Q=-2$ there are generically no zero-modes of the Dirac operator. 
For $I=1 / 2$ we have a rank-2 vector bundle with $Y$ odd and

$$
d(0,0)=\frac{(|Y|+1)(|Y|+5)}{4},
$$

while the index is 15

$$
\nu=(Q+1)(Q+3) .
$$

So $Y=2 Q+1$ when $Q \geq 0$ and $Y=2 Q+7$ when $Q<-4$. For $Q=-1$ or $Q=-3$ there are again generically no zero-modes. $Q=-2$ has $\nu=-1$ and so degeneracy $d(0,0)=1$; it is therefore a singlet of $\mathrm{SU}(2)$ and requires $I=0$ and $Y=0$ : the Fermion spin cancels against the isospin and monopole charge (analogous to $J=1 / 2$ in (2.46)).

\section{Non-symmetric spaces}

On a non-symmetric coset space the analysis of section 2 requires some minor modifications. On non-symmetric spaces there is a natural spin connection which has torsion, as described in appendix A, and equation (2.4) is modified to

$$
(i \not)^{2}=\Delta+\frac{R}{4} \mathbf{1}-\frac{1}{8} R_{\alpha \beta \gamma \delta} \gamma^{\alpha \beta \gamma \delta}-\frac{i}{2} F_{\alpha \beta} \gamma^{\alpha \beta},
$$

because $R_{[\beta \gamma \delta]}^{\alpha} \neq 0$ when there is torsion. Indeed for non-symmetric coset spaces there is a spin connection with

$$
R_{\beta}^{\alpha} \wedge \mathrm{e}^{\beta}=d T^{\alpha}+\omega^{\alpha}{ }_{\beta} \wedge T^{\beta},
$$

where

$$
T^{\alpha}=\frac{1}{2} f_{\beta \gamma}^{\alpha} \mathrm{e}^{\beta} \wedge \mathrm{e}^{\gamma}
$$

are the torsion 2-forms. The connection involving torsion is used here because the resulting curvature tensor

$$
R_{\beta}^{\alpha}=\frac{1}{2} f_{\beta i}^{\alpha} f_{\gamma \delta}^{i} \mathrm{e}^{\gamma} \wedge \mathrm{e}^{\delta}
$$

is co-variantly constant with this connection, as shown in appendix A. This means that at least the first three terms on the right hand side of equation (3.1) are mutually commuting and simultaneously diagonalisable.

Identifying the gauge connection with the spin connection now gives the same expression as for symmetric spaces

$$
F^{i}=\frac{1}{2} f_{\alpha \beta}^{i} \mathrm{e}^{\alpha} \wedge \mathrm{e}^{\beta}
$$

and we see that

$$
R_{\alpha \beta \gamma \delta} \gamma^{\alpha \beta \gamma \delta}=f_{\alpha \beta}^{i} f_{\gamma \delta}^{i} \gamma^{\alpha \beta \delta \gamma}
$$

is related to $\operatorname{tr}(F \wedge F)$. Furthermore

$$
f_{\alpha \beta}^{i} f_{\gamma \delta}^{i} \gamma^{\alpha \beta \delta \gamma}=f_{\alpha \beta}^{i} f_{\gamma \delta}^{i} \gamma^{\alpha \beta} \gamma^{\gamma \delta}+2 f_{\alpha \beta}^{i} f_{\alpha \beta}^{i}=-16 C_{2}\left(H, T_{i}\right)+2 R
$$

commutes with $F_{\alpha \beta}^{i} \gamma^{\alpha \beta}, \mathbf{1}$ and $\Delta$ (the last because $F_{\alpha \beta}^{i}$ is co-variantly constant). Now every term on the right hand side of (3.1) is mutually commuting and it reads

$$
(i \not)^{2}=\Delta+2 C_{2}\left(H, T_{i}\right)-\frac{i}{2} F_{\alpha \beta} \gamma^{\alpha \beta}
$$


Paralleling the argument that led to (2.15) for symmetric spaces the eigenvalues can be expressed terms of Casimirs

$$
E=C_{2}(G, \cdot)-C_{2}\left(H, t_{i}\right)+C_{2}\left(H, T_{i}\right)
$$

This equation is more general than (2.15) and includes it as a special case, because $C_{2}\left(H, T_{i}\right)=R / 8$ for symmetric coset spaces.

As an illustration consider the space $\mathrm{SU}(3) / \mathrm{U}(1) \times \mathrm{U}(1)$, which is of interest in string theory, where a seven dimensional space with $G_{2}$ holonomy can have a conical singularity on $\mathrm{SU}(3) / \mathrm{U}(1) \times \mathrm{U}(1)$, [9]. We shall not calculate the full spectrum of the Dirac operator here, but shall concentrate on the zero-modes. There are two independent $\mathrm{U}(1)$ gauge fields and we can take "monopoles" of both, so the background gauge field is labelled by two integers $Y$ and $T$, which are the hypercharge and the third component of isospin of a $\mathrm{SU}(3)$ irreducible representation. Under

$$
\mathrm{SU}(3) \quad \rightarrow \quad \mathrm{SU}(2) \times \mathrm{U}(1) \quad \rightarrow \quad \mathrm{U}(1) \times \mathrm{U}(1)
$$

The $\mathbf{3}$ of SU(3) decomposes as

$$
\mathbf{3} \quad \rightarrow \quad \mathbf{2}_{1}+\mathbf{1}_{-2} \quad \rightarrow \quad(1,1)+(-1,1)+(0,-2)
$$

Let $₫=(1,1), \square=(-1,1), \square=(0,-2), \bigotimes=(0,2)$. Then $\dot{\otimes}=(1,-1)$ and $\dot{\theta}=(-1,-1)$. The $(p, \bar{p})$ representation of $\mathrm{SU}(3)$ contains

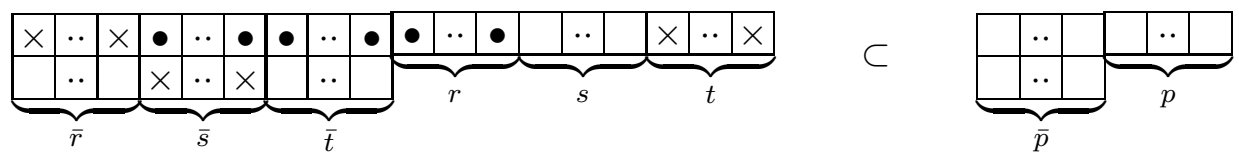

with $r+s+t=p, \bar{r}+\bar{s}+\bar{t}=\bar{p}$. Demanding that the $(p, \bar{p})$ representation of $\mathrm{SU}(3)$ contains the $(T, Y)$ representation of $\mathrm{U}(1) \times \mathrm{U}(1)$ puts constraints on the six integers $(r, s, t ; \bar{r}, \bar{s}, \bar{t})$, namely

$$
Y=(s-\bar{s})+(t-\bar{t})-2(r-\bar{r}) \quad \text { and } \quad T=(t-\bar{t})-(s-\bar{s}) .
$$

The $\mathrm{SU}(3)$ states that contain $(T, Y)$ are therefore labelled by four independent integers (note that $Y$ and $T$ are either both even or both odd). If zero-modes of the Dirac operator exist, then their number is given by the dimension of the $\mathrm{SU}(3)$ representation which minimises the second order Casimir (2.49), subject to these constraints. We find

$$
\begin{aligned}
& T \geq Y \geq 0 \Rightarrow C_{2}^{\min }=T+\frac{T^{2}}{4}+\frac{Y^{2}}{12}, \text { for }(r, s, t ; \bar{r}, \bar{s}, \bar{t})=\left(0,0, \frac{T+Y}{2} ; 0, \frac{T-Y}{2}, 0\right) \\
& Y \geq T \geq 0 \Rightarrow C_{2}^{\min }=\frac{T+Y}{2}+\frac{T^{2}}{4}+\frac{Y^{2}}{12}, \text { for }(r, s, t ; \bar{r}, \bar{s}, \bar{t})=\left(0,0, T ; \frac{Y-T}{2}, 0,0\right) .
\end{aligned}
$$

These two representations have dimensions

$$
d_{\min }=\frac{1}{8}(T+Y+2)(T-Y+2)(T+2) \quad \text { and } \quad d_{\min }=\frac{1}{8}(Y+T+4)(Y-T+2)(T+1)
$$


respectively. Other combinations of signs follow from

$$
\begin{array}{lll}
T \rightarrow-T, Y \rightarrow-Y & \text { interchange } & (r, s, t) \leftrightarrow(\bar{r}, \bar{s}, \bar{t}) \\
T \rightarrow-T, Y \rightarrow Y & \text { interchange } & (s, \bar{s}) \leftrightarrow(t, \bar{t}) \\
T \rightarrow T, Y \rightarrow-Y & \text { interchange } & (r, s, t) \leftrightarrow(\bar{r}, \bar{t}, \bar{s}) .
\end{array}
$$

In summary the dimensions of the ground states are

$$
\begin{aligned}
& |T| \geq|Y| \Rightarrow d_{\min }=\frac{1}{8}\left((|T|+2)^{2}-Y^{2}\right)(|T|+2) \\
& |Y| \geq|T| \Rightarrow d_{\min }=\frac{1}{8}\left((|Y|+3)^{2}-(|T|+1)^{2}\right)(|T|+1) .
\end{aligned}
$$

That these do indeed correspond to zero-modes can be established by comparison with a more standard analysis (as presented in appendix C for example): for U(1) fields with monopole charges $M$ (hypercharge) and $N$ (third component of isospin), the index is (C.8)

$$
\nu=\frac{1}{8} M\left(N^{2}-M^{2}\right) .
$$

Clearly

$$
M=T \pm 2 \quad \text { and } \quad N=Y, \quad \text { for } \quad|T| \geq|Y|
$$

and

$$
M=T \pm 1 \quad \text { and } \quad N=Y \pm 3, \quad \text { for } \quad|Y| \geq|T|
$$

will do the trick (either identification works when $|T|=|Y|$ ). This shift is again due to the intrinsic spin of the Fermions contributing to the $\mathrm{U}(1)$ charges.

\section{Conclusions}

In this paper the spectrum of the Dirac operator, for Fermions coupled to topology nontrivial homogeneous background gauge fields on compact coset spaces $G / H$, has been determined purely in terms of quadratic Casimirs. The spectra are obtained from (3.9), which reduces to (2.15) for symmetric spaces. This is the central result.

It has been shown how the ground state degeneracies encountered in discussions of the quantum Hall effect on $S^{2}$, and its higher dimensional generalisations to $S^{4}$ and $C P^{n}$, are related to the Atiyah-Singer index theorem for spinors on these spaces. Physically the square of the Dirac operator is the non-relativistic hamiltonian for a particle moving in a background "magnetic" field and consists of a kinetic term and a Zeeman splitting term (at least for zero torsion). For spinors on a coset space $G / H$ and gauge group $H,{ }^{1}$ moving in a homogeneous background field identified with the natural spin connection, the kinetic term and the Zeeman splitting term commute. The hamiltonian can then be diagonalised resulting in a set of uncoupled spin states, each of which can be treated independently.

If all we are interested in is zero-modes and the index theorem it is not necessary to compute the whole spectrum. If zero-modes of the Dirac operator exist the ground state must be one in which the Zeeman energy exactly cancels the kinetic energy and its de-

\footnotetext{
${ }^{1}$ Or a factor group if $H$ is a product of Lie groups.
} 
generacy is the number of zero-modes. Since the kinetic energy is positive-definite, this cancellation requires a minimum of the quadratic Casimir corresponding to the kinetic energy. The degeneracy of the ground state is then just the dimension of the representation of $G$ that minimises the quadratic Casimir - subject to the condition that only representations of $G$ that contain the representations of $H$ relevant to the spinor dynamics are considered. Generically the number of zero-modes is the same as the modulus of index of the Dirac operator, so this gives a method of calculating the index just by using knowledge of the quadratic Casimirs.

The construction is not one-to-one: there may be more than one possible representation of the gauge group giving the same number of zero-modes of Dirac operator. For example on $C P^{2}$ an $\mathrm{SU}(2)$ singlet with $Q=-3$ has $|\nu|=1$ which is the same as for an $\mathrm{SU}(2)$ doublet with $Q=-2$. The complete spectrum of the Dirac operator on $C P^{2}$ is given in appendix B.

The technique also works for non-symmetric space with torsion and the example of $\mathrm{SU}(3) / \mathrm{U}(1) \times \mathrm{U}(1)$ has been treated in detail.

\section{Acknowledgments}

It is a pleasure to thank Charles Nash for useful discussions on the Dirac operator and the Atiyah-Singer index theorem.

\section{A. Metric and connection on $G / H$}

In this section we summarise the construction of homogeneous connections and curvatures on coset spaces. None of this material is new: the construction is well known to mathematician's [16]. The development here is based on the exposition for physicists in [18.

On coset spaces $G / H$, with $G$ and $H$ compact Lie groups and $G$ simple, the Riemann tensor can be obtained in terms of the structure constants of $G$. Denote the generators of $G$ by $t_{A}$, with the algebra

$$
\left[t_{A}, t_{B}\right]=i f_{A B}{ }^{C} t_{C}
$$

The set of generators $\left\{t_{A}\right\}$ decomposes into generators of $H$, which shall be denoted by $t_{i}$, and the remaining generators, which will be labelled $t_{\alpha}$. Thus $\alpha$ takes on $d$-values where $d$ is the dimension of $G / H$. The algebra of $G$ now splits up as

$$
\left[t_{i}, t_{j}\right]=i f_{i j}{ }^{k} t_{k} \quad\left[t_{i}, t_{\alpha}\right]=i f_{i \alpha}{ }^{\beta} t_{\beta} \quad\left[t_{\alpha}, t_{\beta}\right]=i f_{\alpha \beta}{ }^{\gamma} t_{\gamma}+i f_{\alpha \beta}{ }^{k} t_{k} .
$$

The space $G / H$ is called symmetric if $f_{\alpha \beta}{ }^{\gamma}=0$, when this is the case the decomposition (A.2) is invariant under $t_{\alpha} \rightarrow-t_{\alpha}$.

Orthonormal 1-forms for the group $G$ can be chosen as the Cartan 1-forms,

$$
g^{-1} d g=i e^{A} t_{A}
$$

SO

$$
d e^{A}=\frac{1}{2} f_{B C}{ }^{A} e^{B} \wedge e^{C} .
$$


Then the subset $e^{\alpha}$ are orthonormal 1-forms for a $G$-invariant metric on $G / H$ and the remaining 1-forms $e^{i}$ can be expanded on $G / H$ as $e^{i}=\Pi_{\alpha}^{i} e^{\alpha}$.

The torsion free $\mathrm{H}$-valued ${ }^{2}$ connection $\omega^{\alpha}{ }_{\beta}$ is then defined by

$$
d e^{\alpha}+\omega^{\alpha}{ }_{\beta} \wedge e^{\beta}=0
$$

and evaluates to

$$
\omega_{\beta}^{\alpha}=\left(\frac{1}{2} f_{\beta \gamma}^{\alpha}+f_{\beta i}^{\alpha} \Pi_{\gamma}^{i}\right) e^{\gamma} .
$$

The curvature 2-forms can then be calculated from

$$
R_{\beta}^{\alpha}=d \omega_{\beta}^{\alpha}+\omega_{\gamma}^{\alpha} \wedge \omega_{\beta}^{\gamma}
$$

resulting in

$$
R_{\beta}^{\alpha}=\frac{1}{4}\left(2 f_{\beta i}^{\alpha} f_{\gamma \delta}^{i}+f_{\beta \epsilon}^{\alpha} f_{\gamma \delta}^{\epsilon}-f_{\gamma \epsilon}^{\alpha} f_{\beta \delta}^{\epsilon}\right) e^{\gamma} \wedge e^{\delta} .
$$

On a symmetric space these reduce to the simpler form

$$
R_{\beta}^{\alpha}=\frac{1}{2}\left(f_{\beta i}^{\alpha} f_{\gamma \delta}^{i}\right) e^{\gamma} \wedge e^{\delta},
$$

so the Riemann tensor has components

$$
R_{\beta \gamma \delta}^{\alpha}=f_{\beta i}^{\alpha} f_{\gamma \delta}^{i} .
$$

On a non-symmetric space there is a second, very useful, connection that comes from introducing a torsion tensor which is identified with the non-symmetric structure constants:

$$
T_{\beta \gamma}^{\alpha}=f_{\beta \gamma}^{\alpha}
$$

giving torsion 2-forms

$$
T^{\alpha}=\frac{1}{2} f_{\beta \gamma}^{\alpha} e^{\beta} \wedge e^{\gamma}
$$

Then the connection with torsion is defined via

$$
d e^{\alpha}+\omega_{\beta}^{\alpha} \wedge e^{\beta}=T^{\alpha}
$$

which leads to

$$
\omega_{\beta}^{\alpha}=\frac{1}{2} f^{\alpha}{ }_{\beta i} \Pi_{\gamma}^{i} e^{\gamma} .
$$

The resulting curvature 2 -forms are

$$
R_{\beta}^{\alpha}=\frac{1}{2} f_{\beta i}^{\alpha} f_{\gamma \delta}^{i} e^{\gamma} \wedge e^{\delta},
$$

giving curvature tensor

$$
R_{\beta \gamma \delta}^{\alpha}=f_{\beta i}^{\alpha} f_{\gamma \delta}^{i} .
$$

\footnotetext{
${ }^{2}$ For notational simplicity we do not distinguish between the group and the algebra here.
} 
The Ricci scalar for the connection with torsion is then easily evaluated as

$$
R=R_{\alpha \beta}^{\alpha \beta}=f^{\alpha \beta}{ }_{i} f_{\alpha \beta}=f^{A B}{ }_{i} f_{A B}^{i}-f^{j k}{ }_{i} f^{i}{ }_{j k},
$$

which can be determined using the appropriate quadratic Casimirs of $H$.

A particular instance of this is when $H$ is trivial so $G / H \cong G$. Then $\omega^{\alpha}{ }_{\beta}=0$ and $R^{\alpha}{ }_{\beta}=0$, all co-variant derivatives are trivial and $T^{\alpha}$ is called the parallelising torsion for $G$. On a symmetric space, of course, A.8) and (A.15) are identical because $f^{\alpha}{ }_{\beta \gamma}=0$.

In fact it is not difficult to show, using (A.14), A.15) and the Jacobi identity, that $R_{\beta \gamma \delta}^{\alpha}$ in $(\widehat{A .16})$ is co-variantly constant,

$$
\nabla_{\epsilon} R_{\beta \gamma \delta}^{\alpha}=0
$$

On a generic $d$-dimensional manifold the curvature 2-forms (A.7) are $\mathrm{SO}(d)$ Lie algebra valued 2-forms, but on $G / H$ both (A.8) and (A.15) are $\mathrm{H}$ valued 2-forms, where $H \subseteq \mathrm{SO}(d)$. This means that we can take linear combinations of (A.15) that lie in $\mathrm{H}$ without losing any information. For example, if $H$ is semi-simple, taking the combination

$$
f_{\alpha \beta}^{i} R^{\alpha \beta}=\frac{1}{2}\left(C_{2}(G, a d j)-C_{2}(H, a d j)\right) f_{\gamma \delta}^{i} \mathrm{e}^{\gamma} \wedge \mathrm{e}^{\delta}
$$

suggests defining

$$
F^{i}:=\frac{1}{2} f_{\gamma \delta}^{i} e^{\gamma} \wedge e^{\delta}
$$

and then $F^{i}$ are $\mathrm{H}$-valued 2-forms which are equivalent to (A.15) (this formula is easily adapted to the case where $H$ contains $\mathrm{U}(1)$ factors).

\section{B. Spectrum of the Dirac operator on $C P^{2}$}

The calculation of the full spectrum of the Dirac operator on $C P^{2}$ proceeds as follows (the spectrum on $C P^{n}$, with $n$ odd and no background gauge field, has been considered in [17]). For $\mathrm{SU}(3)$ the second order Casimir and dimension are

$$
C_{2}(p, \bar{p})=\frac{1}{3}(p(p+3)+\bar{p}(\bar{p}+3)+p \bar{p})
$$

and

$$
d(p, \bar{p})=\frac{1}{2}(p+\bar{p}+2)(p+1)(\bar{p}+1)
$$

respectively. With $p=r+s$ and $\bar{p}=\bar{r}+\bar{s}$, as in the text, the constraints read

$$
(s-\bar{s})-2(r-\bar{r})=Y \quad \text { and } \quad \frac{s+\bar{s}}{2}=I,
$$

where $Y$ is even (odd) for $I$ integral (half-integral). Now the spectrum depends on whether $|Y| \geq 2 I$ or $|Y| \leq 2 I$ : 
- If $Y \geq 2 I$ then $\bar{r} \geq r$ : in this case let $n=\bar{s}$, so $n=0, \ldots, 2 I, r=k$ and $\bar{r}=$ $k+n-I+\frac{Y}{2}$, for $k$ a non-negative integer. If $Y \leq-2 I$ then $r \geq \bar{r}$ : in this case let $n=s$, so $n=0, \ldots, 2 I, r=k+n-I-\frac{Y}{2}$ and $\bar{r}=k$, for $k$ a non-negative integer. In either case

$C_{2}(p, q)=k\left(k+n+2+I+\frac{|Y|}{2}\right)+n\left(n+1-I+\frac{|Y|}{2}\right)+\frac{|Y|}{2}+\frac{Y^{2}}{12}+I(I+1)$.

For $C_{2}\left(H, t_{i}\right)$ in (2.15) take the $\mathrm{U}(1)$ background to have fixed charge $M$ and the $\mathrm{SU}(2)$ background to have isospin $J$, so

$$
C_{2}\left(H, t_{i}\right)=\frac{M^{2}}{12}+J(J+1)
$$

(the $\frac{1}{12}$ here is because the $\mathrm{U}(1)$ gauge field is a multiple of $\frac{1}{2 \sqrt{3}}$ to conform with the normalisation of $t_{8}$ in appendix CD. Finally the Ricci scalar for $C P^{2}$ can be evaluated from (A.17) and the structure constants in appendix $\mathrm{C}$ to be $R=6$ so, putting all this together, the eigenvalues of (2.15) are

$$
\begin{aligned}
E(k, n)= & k\left(k+n+2+I+\frac{|Y|}{2}\right)+n\left(n+1-I+\frac{|Y|}{2}\right)+ \\
& +\frac{(|Y|+3)^{2}}{12}-\frac{M^{2}}{12}+I(I+1)-J(J+1),
\end{aligned}
$$

while the degeneracies are

$$
d(k, n)=\frac{1}{2}\left(2 k+n+I+2+\frac{|Y|}{2}\right)\left(k+2 n-I+1+\frac{|Y|}{2}\right)(k-n+2 I+1),
$$

with $n=0, \ldots, 2 I$ and $k \geq 0$ an integer.

It is important to understand how the gauge charges $M$ and $J$ are related to the total charges $Y$ and $I$ (which include the spin connection). There are four cases to consider:

1. $M=Y \pm 3$ and $I=J$, these are states that couple to the $\mathrm{U}(1)$ part of the spin connection and not the $\mathrm{SU}(2)$ part (the \pm 3 relates to the fact that the first Chern class of the tangent bundle for $C P^{2}$ is 3 ). The spectrum is

$$
E(k, n)=k\left(k+n+2+I+\frac{|Y|}{2}\right)+n\left(n+1-I+\frac{|Y|}{2}\right)+\frac{|Y| \mp Y}{2} ;
$$

For $\mathrm{SU}(2)$ singlets $I=J=0$, so $n=0$, this spectrum agrees with the results of [19] (in the notation of that reference $M=2 m+3$, so $Y / 2=m$ or $m+3$ ).

2. $M=Y$ and $I=J \pm \frac{1}{2}$, these are states that couple to the $\mathrm{SU}(2)$ part of the spin connection and not the $\mathrm{U}(1)$ part. The spectrum is

$$
\begin{aligned}
& E(k, n)=k\left(k+n+2+I+\frac{|Y|}{2}\right)+n\left(n+1-I+\frac{|Y|}{2}\right)+\frac{|Y|}{2}+I+1 ; \\
& E(k, n)=k\left(k+n+2+I+\frac{|Y|}{2}\right)+n\left(n+1-I+\frac{|Y|}{2}\right)+\frac{|Y|}{2}-I .
\end{aligned}
$$


For $|Y|>2 I$ only case 1 above allows for zero-modes (when $k=n=0$ ). For $|Y|=2 I$ there are zero-modes in both cases.

- If $0 \leq Y \leq 2 I$, let $n=\frac{\bar{s}-s+Y}{2}$, so $n=-I+Y / 2, \ldots, I+Y / 2$. Then: either $r=k+|n|$ and $\bar{r}=k$; or $r=k$ and $\bar{r}=k+|n|$. If $-2 I \leq Y \leq 0$, let $n=\frac{s-\bar{s}-Y}{2}$, so $n=-I-Y / 2, \ldots, I-Y / 2$. Then: either $r=k+|n|$ and $\bar{r}=k$; or $r=k$ and $\bar{r}=k+|n|$. In either case:

$$
C_{2}(p, q)=k(k+2 I+2)+n^{2}+|n|(k+I+1)-\frac{n}{2}|Y|+\frac{Y^{2}}{12}+I^{2}+2 I .
$$

The eigenvalues of (2.15) are therefore

$$
\begin{aligned}
E(k, n)= & k(k+2 I+2)+n^{2}+|n|(k+I+1)-\frac{n}{2}|Y|+ \\
& +\frac{Y^{2}-M^{2}}{12}+I^{2}+2 I-J(J+1)+\frac{3}{4},
\end{aligned}
$$

with degeneracies

$$
d(k, n)=\left\{(k+I+1)^{2}+|n|(k+I+1)-\frac{(4 n-|Y|)(2 n-|Y|)}{4}\right\}\left(k+I+1+\frac{|n|}{2}\right),
$$

where $-I+\frac{|Y|}{2} \leq n \leq I+\frac{|Y|}{2}$ and $k \geq 0$. (The degeneracy is always an integer because of the restriction that $Y$ is odd when $I$ is half-integral and even if $I$ is integral.)

Again there are four possibilities:

1. $M=Y \pm 3$ and $I=J$,

$$
E(k, n)=k(k+2 I+2)+n^{2}+|n|(k+I+1)-\frac{n|Y|}{2}+I \mp \frac{Y}{2} ;
$$

2. $M=Y$ and $I=J \pm \frac{1}{2}$,

$$
\begin{aligned}
& E(k, n)=k(k+2 I+2)+n^{2}+|n|(k+I+1)-\frac{n}{2}|Y|+2 I+1 ; \\
& E(k, n)=k(k+2 I+2)+n^{2}+|n|(k+I+1)-\frac{n}{2}|Y| .
\end{aligned}
$$

These eigenvalues are bounded below by zero and, for $|Y|<2 I$, only (B.16) allows for zero-modes (when $k=n=0$ ). When $|Y|=2 I$ the spectrum agrees with equations (B.8)-(B.10).

\section{Index theorem on $\mathrm{SU}(3) / \mathrm{U}(1) \times \mathrm{U}(1)$}

In this section we give an explicit evaluation of the index of the Dirac operator on $\mathrm{SU}(3) / \mathrm{U}(1) \times \mathrm{U}(1)$, using standard differential-geometric techniques.

Let $\lambda_{A} ; A=1, \ldots, 8$ be the Gell-Mann matrices for $\mathrm{SU}(3)$, so

$$
\left[t_{A}, t_{B}\right]=i f_{A B}^{C} t_{C} \quad \text { with } \quad t_{A}=\frac{\lambda_{A}}{2}
$$


and

$$
f_{123}=1, \quad f_{453}=-f_{673}=f_{471}=-f_{561}=f_{462}=f_{572}=\frac{1}{2}, \quad f_{458}=f_{678}=\frac{\sqrt{3}}{2} .
$$

In the notation of appendix $\mathrm{A}, i=3,8$ and $\alpha=1,2,4,5,6,7$, when

$$
t_{3}=\frac{1}{2}\left(\begin{array}{ccc}
1 & 0 & 0 \\
0 & -1 & 0 \\
0 & 0 & 0
\end{array}\right) \quad \text { and } \quad t_{8}=\frac{1}{2 \sqrt{3}}\left(\begin{array}{ccc}
1 & 0 & 0 \\
0 & 1 & 0 \\
0 & 0 & -2
\end{array}\right)
$$

are chosen as the $\mathrm{U}(1) \times \mathrm{U}(1)$ generators. This space is not symmetric, because some of the $f_{\alpha \beta \gamma} \neq 0$. The curvature 2 -forms, for the spin connection with torsion described in appendix A, are

$$
\begin{aligned}
& R_{12}=\frac{1}{2}\left(2 \mathrm{e}^{1} \wedge \mathrm{e}^{2}+\mathrm{e}^{4} \wedge \mathrm{e}^{5}-\mathrm{e}^{6} \wedge \mathrm{e}^{7}\right) \\
& R_{45}=\frac{1}{2}\left(\mathrm{e}^{1} \wedge \mathrm{e}^{2}+2 \mathrm{e}^{4} \wedge \mathrm{e}^{5}+\mathrm{e}^{6} \wedge \mathrm{e}^{7}\right) \\
& R_{67}=\frac{1}{2}\left(-\mathrm{e}^{1} \wedge \mathrm{e}^{2}+\mathrm{e}^{4} \wedge \mathrm{e}^{5}+2 \mathrm{e}^{6} \wedge \mathrm{e}^{7}\right) .
\end{aligned}
$$

These are not independent, since $R_{45}=R_{12}+R_{67}$, they are associated with two U(1) field strengths:

$$
\begin{aligned}
& F^{3} t_{3}=\frac{1}{2} f_{\alpha \beta}{ }^{3}\left(\mathrm{e}^{\alpha} \wedge \mathrm{e}^{\beta}\right) t_{3}=\frac{1}{4}\left(2 \mathrm{e}^{1} \wedge \mathrm{e}^{2}+\mathrm{e}^{4} \wedge \mathrm{e}^{5}-\mathrm{e}^{6} \wedge \mathrm{e}^{7}\right)\left(\begin{array}{ccc}
1 & 0 & 0 \\
0 & -1 & 0 \\
0 & 0 & 0
\end{array}\right) \\
& F^{8} t_{8}=\frac{1}{2} f_{\alpha \beta}{ }^{8}\left(\mathrm{e}^{\alpha} \wedge \mathrm{e}^{\beta}\right) t_{8}=\frac{1}{4}\left(\mathrm{e}^{4} \wedge \mathrm{e}^{5}+\mathrm{e}^{6} \wedge \mathrm{e}^{7}\right)\left(\begin{array}{ccc}
1 & 0 & 0 \\
0 & 1 & 0 \\
0 & 0 & -2
\end{array}\right) .
\end{aligned}
$$

We extract $\mathrm{U}(1)$ singlets by projecting out the top left-hand components

$$
\begin{aligned}
& F_{(3)}=\frac{1}{4}\left(2 \mathrm{e}^{1} \wedge \mathrm{e}^{2}+\mathrm{e}^{4} \wedge \mathrm{e}^{5}-\mathrm{e}^{6} \wedge \mathrm{e}^{7}\right) \\
& F_{(8)}=\frac{1}{4}\left(\mathrm{e}^{4} \wedge \mathrm{e}^{5}+\mathrm{e}^{6} \wedge \mathrm{e}^{7}\right) .
\end{aligned}
$$

A monopole field with charges $(M, N)$ is a linear combination of these

$$
F=M F_{(3)}+N F_{(8)}
$$

from which

$$
F \wedge F \wedge F=\frac{3}{16} M\left(N^{2}-M^{2}\right) \mathrm{e}^{124567}
$$

(we use the shorthand $\mathrm{e}^{124567}=\mathrm{e}^{1} \wedge \mathrm{e}^{2} \wedge \mathrm{e}^{4} \wedge \mathrm{e}^{5} \wedge \mathrm{e}^{6} \wedge \mathrm{e}^{7}$ ). The index of the Dirac operator is 20]

$$
\nu=\frac{1}{(2 \pi)^{3}} \int\left\{\frac{1}{6} F \wedge F \wedge F+\frac{1}{48} F \wedge t r(R \wedge R)\right\} .
$$


Explicit calculation reveals that $F \wedge \operatorname{tr}(R \wedge R)=0$, so

$$
\nu=\frac{1}{256 \pi^{3}} M\left(N^{2}-M^{2}\right) V,
$$

where $V=\int \mathrm{e}^{124567}$ is the volume of $\mathrm{SU}(3) / \mathrm{U}(1) \times \mathrm{U}(1)$. The normalisation can be fixed by using the fact $\mathrm{SU}(3) / \mathrm{U}(1) \times \mathrm{U}(1)$ has Euler characteristic $\chi=6$, so

$$
\chi=\frac{1}{3 !} \frac{1}{(4 \pi)^{3}} \int \epsilon^{\alpha_{1} \cdots \alpha_{6}} R_{\alpha_{1} \alpha_{2}} \wedge R_{\alpha_{3} \alpha_{4}} \wedge R_{\alpha_{5} \alpha_{6}}=6 .
$$

this fixes $V=32 \pi^{2}$ so

$$
\nu=\frac{1}{8} M\left(N^{2}-M^{2}\right) .
$$

Note that $M$ and $N$ must be either both even or both odd for $\nu$ to be an integer.

\section{References}

[1] S.M. Girvin, The quantum hall effect: novel excitations and broken symmetries, cond-mat/9907002.

[2] B.A. Bernevig, J.H. Brodie, L. Susskind and N. Toumbas, How bob laughlin tamed the giant graviton from taub-nut space, J. High Energy Phys. 02 (2001) 003 hep-th/0010105.

[3] S.-C. Zhang and J.-p. Hu, A four dimensional generalization of the quantum hall effect, Science 294 (2001) 823 cond-mat/0110572;

J.-p. Hu and S.-C. Zhang, Collective excitations at the boundary of a 4 D quantum hall droplet, cond-mat/0112432.

[4] F.D.M. Haldane, Fractional quantization of the hall effect: a hierarchy of incompressible quantum fluid states, Phys. Rev. Lett. 51 (1983) 605.

[5] Y.-X. Chen, B.-Y. Hou and B.-Y. Hou, Non-commutative geometry of 4-dimensional quantum hall droplet, Nucl. Phys. B 638 (2002) 220 hep-th/0203095;

B.A. Bernevig, C.-H. Chern, J.-P. Hu, N. Toumbas and S.-C. Zhang, Effective field theory description of the higher dimensional quantum hall liquid, Ann. Phys. (NY) 300 (2002) 185 cond-mat/0206164;

H. Elvang and J. Polchinski, The quantum hall effect on $R^{4}$, hep-th/0209104;

B.-Y. Hou and D.-T. Peng, Incompressible quantum hall fluid, hep-th/0210173.

[6] D. Karabali and V.P. Nair, Quantum hall effect in higher dimensions, Nucl. Phys. B 641 (2002) 533 hep-th/0203264.

[7] M. Fabinger, Higher-dimensional quantum hall effect in string theory, J. High Energy Phys. 05 (2002) 037 hep-th/0201016.

[8] F. Cooper, A. Khare and U. Sukhatme, Supersymmetry and quantum mechanics, Phys. Rept. 251 (1995) 267 hep-th/9405029.

[9] M. Atiyah and E. Witten, M-theory dynamics on a manifold of $G_{2}$ holonomy, Adv. Theor. Math. Phys. 6 (2003) 1 hep-th/0107177.

[10] A.P. Balachandran, G. Immirzi, J. Lee and P. Prešnajder, Dirac operators on coset spaces, hep-th/0210297. 
[11] T. Eguchi, P. Gilkey and A. Hanson, Gravitation, gauge theories and differential geometry, Phys. Rept. 66 (1980) 213

[12] Chen Ning Yang, Generalization of dirac's monopole to SU(2) gauge fields, J. Math. Phys. 19 (1978) 320; SU(2) monopole harmonics, J. Math. Phys. 19 (1978) 2622

[13] A.A. Belavin, A.M. Polyakov, A.S. Shvarts and Y.S. Tyupkin, Pseudoparticle solutions of the Yang-Mills equations, Phys. Lett. B 59 (1975) 85.

[14] S.W. Hawking and C.N. Pope, Generalized spin structures in quantum gravity, Phys. Lett. B $73(1978) 42$.

[15] B.P. Dolan and C. Nash, Chiral fermions and $\operatorname{Spin}^{c}$ structures on matrix approximations to manifolds, J. High Energy Phys. 07 (2002) 057 hep-th/0207007.

[16] S. Kobayashi and K. Nomizu, Foundations of differential geometry, Interscience Publishers, New York 1963.

[17] M. Cahen, A. Franc and S. Gutt, Spectrum of Dirac operator on complex projective spaces $P_{2 q-1}(\mathbf{C})$, Lett. Math. Phys. 18 (1989) 165, erratum ibid. 32 (1994) 365;

S. Seifarth and U. Semmelmann, The spectrum of the Dirac operator on odd dimensional complex projective spaces $P^{2 m-1}(\mathbf{C})$, SFB 288 preprint 95 (1993);

C. Bär, Metrics and harmonic spinors, Geometry and functional analysis, 6 (1996) 899.

[18] A. Salam and J. Strathdee, On Kaluza-Klein theory, Ann. Phys. (NY) 141 (1982) 316.

[19] H. Grosse and A. Strohmaier, Noncommutative geometry and the regularization problem of $4 d$ quantum field theory, Lett. Math. Phys. 48 (1999) 163 hep-th/9902138].

[20] L. Alvarez-Gaumé and P. Ginsparg, The structure of gauge and gravitational anomalies, Ann. Phys. (NY) 161 (1985) 423, erratum ibid. 171 (1986) 233. 